\title{
10 Satellite-Based Remote Sensing
}

\subsection{Introduction}

Those beginning to read the book at this chapter could find it troublesome with so many references to previous chapters, but it is the only way we found to make this chapter both short and straightforward. We think that these references will also help the readers to refresh the needed concepts that they might have forgotten since they read the first chapters.

We will focus this chapter on the technical characteristics of the instruments on-board satellites, avoiding any reference to the way in which the geometric aspects of the flight determine the composition of the images. Nor are we going to treat how images have to be interpreted. These subjects can be found in most books on remote sensing (Campbell, 2007; Lillesand et al., 2004). As in the rest of the book our interest will be centered on how instruments work in order to understand their applications and limitations.

\subsection{Preliminary Discussion}

Satellite-based remote sensing is a vast and rapidly changing field. Battery degradation, unexpected failures and exhaustion of propellants, among others, are problems that make satellites' life quite limited. For example, geosynchronous satellites require propellants to keep them in orbit and to maintain their altitude so that the solar panels and antenna can be pointed adequately. Therefore, because they run out of propellant, the useful lifetime of geosynchronous satellites averages about fifteen years (Kurtin, 2013). Also, some missions with polar orbits planned for several years and carrying sophisticated remote sensing equipment lasted less than two years due to failure in the energy system or in the instruments (http://coaps.fsu. edu/scatterometry/about/overview.php).

Similar problems are faced by communication satellites (Section (9.6.4.1)).

The short operating life of satellites, along with the rapid changes in electronics and satellite technologies, makes remote sensing from space a very dynamic field. There are many different instruments working on board spacecrafts, as well as many names, e.g. active microwave instruments, synthetic aperture radar, wind scatterometer, radar altimeter, scanning radiometer, ozone monitor, microwave sounder, precise range and range rate equipment, laser reflector, microwave radiometer, LIDAR, precise orbit Doppler locator, laser tracker, GPS tracker, precipitation radar, microwave imager, visible infrared scanner, clouds and Earth's radiant energy system, lighting imaging sensor, etc. 
For inexperienced researchers willing to start in the satellite instrumentation field, the problem looks very confusing because they observe instruments with similar operating principles and different names. But from the point of view of the experts, these instruments are really very different due to the specific characteristics that allow them to gather different information from the same piece of the Earth's surface. For this reason, experts create new names to underline the differences from former instruments, even though the operating principles are quite similar.

It is difficult to describe in a book all the instruments flying in one modern satellite. Besides, due to the fast changes in remote sensing strategies any description runs the risk of becoming quickly obsolete. For these reasons, only lasting concepts on the technologies used in remote sensing and general operating principles will be addressed.

In our efforts to synthesize these issues we will make some oversimplifications. We hope that we will have the comprehension of readers who know about this subject, because they could consider these approximations somewhat rough.

\subsection{Introductory Concepts}

Some concepts on satellites have already been introduced in Section (9.6.4) when satellite communications were explained. The spacecrafts used for remote sensing have many similarities with those described first. They are space platforms whose main differences are the payloads they carry. In some cases there are also some differences in the orbits they travel. Instead of carrying communication equipment, satellites used in remote sensing applications transport instruments for sensing the electromagnetic energy coming from the Earth's surface.

From the point of view of the measured energy, instruments onboard satellites can be differentiated into two major groups: passive and active instruments. In the first group are those instruments that measure the sun energy reflected or re irradiated by the Earth's surface. In the second, instruments that measure the energy sent by them and scattered by the Earth's surface.

Some passive remote sensing instruments gather information in the wavelength range from tenths to tens of micrometers. Usually, instruments designed for this range are tailored either for information in the visible spectra or in the infrared range of the spectra. The latter are known as thermal radiometers or scanners.

There are other instruments that work in the microwave band. Whereas thermal radiometers work with wavelengths about $10 \mu \mathrm{m}$, microwave radiometers work with wavelengths between $1 \mathrm{~mm}$ and $1 \mathrm{~m}$. We find it convenient to clarify this point because the word microwave could be misleading.

Active remote instruments, instead, are similar to radar (Section (8.2)) or lidar (Section (8.8)). A well known selected electromagnetic perturbation is emitted from the instrument towards a target and the backscattered signal is analyzed to obtain information about the target illuminated by the perturbation. Active remote sensing in the radio frequency range 
uses wavelengths on the order of centimeters. Lidar are active devices in the wavelength range from about $10 \mu \mathrm{m}$ (near infrared) to about $250 \mathrm{~nm}$ (ultraviolet).

The different possible types of satellite orbits were already introduced in Section (9.6.4). Many remote sensing spacecrafts follow north-south orbits which, for an observer fixed to the Earth, appear as inclined with respect to the north-south direction due to the Earth's rotation (Coriolis force, Section (3.10)); they are called near-polar orbits. The usual altitude for meteorological satellites is about $900 \mathrm{~km}$ but, as it was explained in Section (9.6.4), geosynchronous satellites need to be at $35,800 \mathrm{~km}$, either if they are used for communication or for metrological purposes. Because the geosynchronous satellites are above the Equator, they do not cover high latitude areas. Satellites in polar orbit provide a frequent coverage of the Polar Regions.

A particular characteristic of some remote sensing satellites not observed in communication satellites is that their near-polar orbit is sun-synchronous. Thus, satellites always cover the same area of the world at the same local time, ensuring similar illumination conditions. Images having the same illumination are easier to compare and changes in one specific area are readily appreciated.

In Section (9.6.4) it was called as footprint the area of the Earth's surface with which a satellite can communicate while following its orbit. In remote sensing field a similar concept, known as swath, is used to define the portion of the Earth's surface that the sensor "sees"; Figure 10.1 depicts a satellite and its swath. The Earth's rotation determines that in each orbit the instrument on board the satellite cover a new swath, thus the complete Earth's surface is imaged after some regular time.

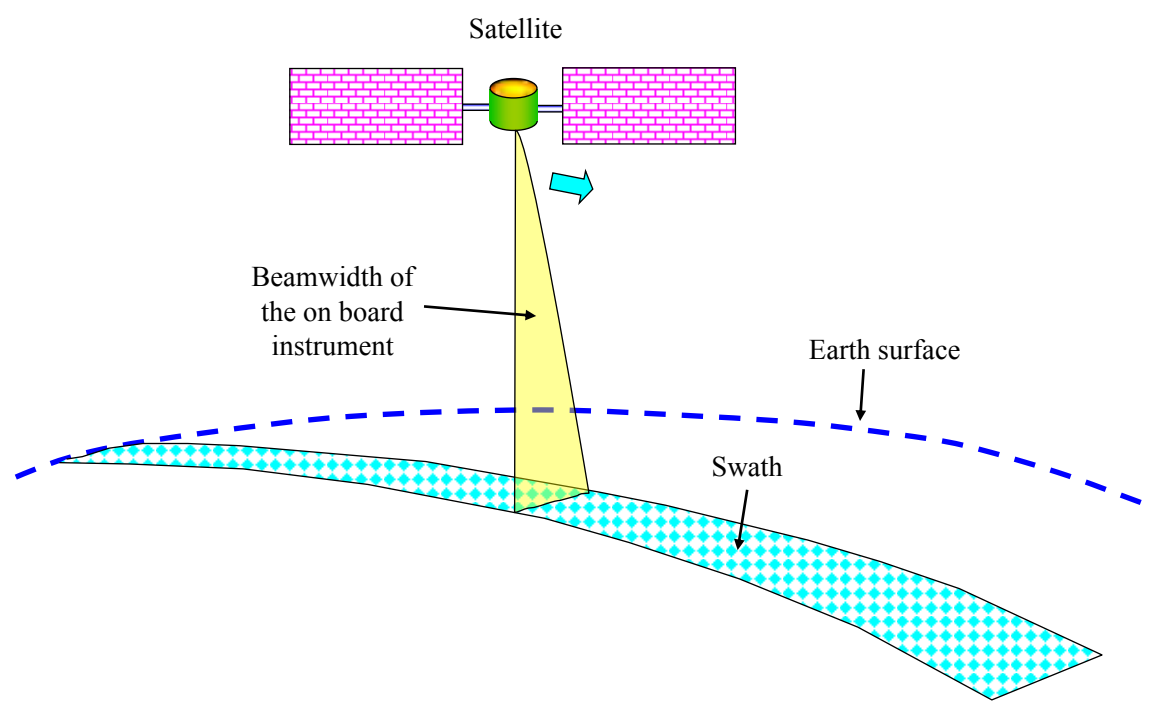

Fig. 10.1: The beamwidth of the instrument and the orbit of the satellite define a swath on the Earth's surface. 
Figure 10.2 shows the nomenclature used in the geometry of satellite's radar. The angle between the radar beam direction and a line directed towards the nadir is called the look angle. Recall that the nadir is the point where a descendent line oriented perpendicular to the Earth's surface and passing through the satellite and the center of the Earth seems to intercept the celestial sphere (the opposite, upwards point, is the zenith). The complement of the look angle is the depression angle. The incident angle is the angle between the radar beam direction and the normal to the Earth's surface at the point where the radar beam hits the surface (Lillesand et al., 2004).

Satellites devoted to acquiring images of the Earth can be classified into two large groups according to their uses; some are designed to study the Earth's resources, while others are devoted to study environmental phenomena.

Satellite

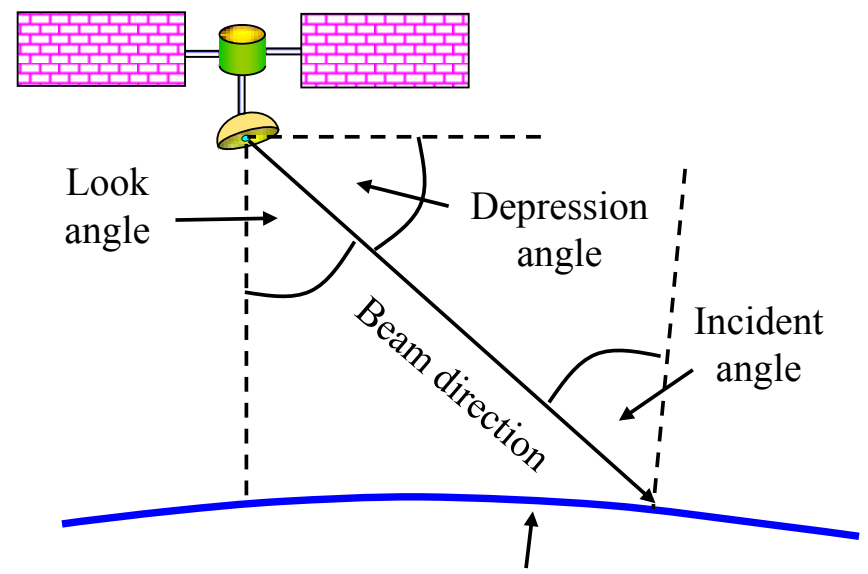

Earth surface

Fig. 10.2: Nomenclature used in satellite's geometry

Earth's resources such as crops do not change very fast, but to estimate the amount and degree of growth it is necessary to have good spatial resolution. Therefore, the swath width of these images is less than $200 \mathrm{~km}$, and the spatial resolution is less than $100 \mathrm{~m}$ (Sabins, 1999). Due to the relative small width of the swaths, these satellites require several days to obtain a complete image of the Earth.

Environmental satellites, in contrast, need to collect information on the complete Earth's surface daily or hourly. Thus, swaths are of hundreds to thousands of kilometers and the spatial resolution of several hundreds of meters (Sabins, 1999). Once again, the concept of spatial and time resolutions of the instruments dedicated to measure environmental parameters is essential in the quality of the data gathered; thus, the concept of image resolution is introduced below. 


\subsection{Image Resolution}

The image resolution gives an indication of the ability of the instruments to capture the Earth's surface information with a certain degree of detail. When speaking of satellite images, in addition to the spatial resolution already mentioned, it is usual to define the terms: spectral resolution, radiometric resolution and temporal resolution. All of them will be described below (http://concurso.cnice.mec.es/cnice2006/material121/ unidad1/i_resolucion.htm).

\subsubsection{Spatial Resolution}

In Section (4.7.7), where infrared thermography was described, we mentioned that the cameras detect the energy emitted from individual points of an object and convert them into a visual image. An image consists of a matrix of pixels; the number of pixels depends on the number of detectors (optical sensors) that the camera has. For example, some cameras for domestic use have $1600 \times 1200$ pixels $\left(1.92 \times 10^{6}\right.$ pixels $)$. The amount of pixels defines the maximum spatial resolution of the camera (the minimum point of the image that can be distinguished). For instance, if the camera flying on board a spacecraft has $4000 \times 3000$ pixels and the swath is $40 \mathrm{~km} \times 30 \mathrm{~km}$, the spatial resolution would be one hundred square meters.

In active remote sensing instruments (radar, lidar, etc) it is more complex to quantify the spatial resolution because it depends on several factors: the beamwidth (linked to the transmitted frequency and antenna), the pulse length and the data processing.

\subsubsection{Spectral Resolution}

Spectral resolution is the amount of spectral channels through which the instrument on board the spacecraft detects the electromagnetic energy coming from the Earth's surface. Different kinds of objects on the Earth's surface can present different spectral responses when illuminated by the sun. Therefore, studying the surface response over distinct wavelength ranges gives information about what objects there are, e.g. to differentiate soil surfaces it is needed to measure on several narrow wavelength ranges.

Some radiometers on board satellites are called multi-spectral sensors because they record energy over a few separate wavelength ranges. For example, the SPOT 6 satellite has a spectral resolution of five, because it records the following wavelengths: panchromatic (450 - $745 \mathrm{~nm})$, blue $(450-520 \mathrm{~nm})$, green $(530-590 \mathrm{~nm})$, red $(625$ - $695 \mathrm{~nm}$ ) and near-infrared (760 - $890 \mathrm{~nm}$ ) (Astrium GeoInformation Services, 2013). More recent radiometers can record hundreds or narrow spectral bands called hyperspectral sensors. Obviously, this high spectral discrimination allows acquiring more information about the targets. 
Active instruments use few frequencies to "sense" the targets, and then we could say that they have a reduced spectral resolution. The electronics and the transmitting and receiving antennas define the spectral resolution.

\subsubsection{Radiometric Resolution}

The radiometric resolution is the ability to discriminate very slight differences in the amount of the received energy. Each optical sensor, which defines a pixel in the image, receives the electromagnetic energy from the Earth's surface and produces a certain analog output, which has to be converted into a digital one. The brightness of each pixel is discretized according to the analog-to-digital converter (Section (3.6.6)) used by the on-board instrument. For example, some satellites had 8 bit resolution, whereas others had 10 bit; i.e. the brightness was converted to 256 or 1024 levels of gray, respectively. In hyperspectral radiometers this discretization is done for each spectral band, thus increasing considerably the amount of resources needed to store or transmit the data.

\subsubsection{Temporal Resolution}

Temporal resolution in satellite vocabulary refers to the period of time needed for a satellite to sense again the same area. The time that it takes for a satellite to complete one entire orbit cycle is called the revisit period; it is the period for the satellite to pass again over exactly the same swath. Because some satellites have the ability to change the pointing angle of their optical systems and antennas, the same area could be "viewed" from another orbit when the satellite travels in a near, but different, orbit. Thus, some satellite can define a temporal resolution smaller than the revisit period. It has to be emphasized that they sense the same region but with a different viewing angle. High resolution satellites have small swaths and a long revisit period, e.g. satellites with a swath $60 \mathrm{~km}$ wide could pass over the same point every 26 days.

\subsection{Instrument's Scanning Geometry}

\subsubsection{Introduction}

It is worth recalling that instruments devoted to study the Earth's surface from on board satellites only measure electromagnetic waves coming from it. Therefore, the purpose of the scanning systems is to point the instrument towards some specific spot on the surface (ground resolution cell). They are merely pointing systems (optomechanical devices) conceived to select pieces of the surface over which the measure will be made. They are designed having in mind how the composition of these pieces (spots) will result in an image of a region of the Earth. 
In order to simplify our explanation we will describe these scanning geometries associated to a passive or active instrument, but the way the Earth's surface is scanned is independent of the instrument itself. They can be used either to point a passive or an active sensor.

\subsubsection{Across-Track Scanning}

In passive instruments there are different ways in which the electromagnetic signal coming from the Earth's surface is conveyed to the sensor through the optical arrangement. One of them is called across-track scanning and consists of a rotating mirror that scans the terrain on the swath in a direction perpendicular to the satellite track. Thus, it measures the energy from one side of the swath to the other traveling an arc, e.g. $90^{\circ}$. As the satellite advances in its trajectory, a new scan is performed. Successive scans form a bi-dimensional image (Fig. 10.3). It has to be emphasized that in this geometry there is only one point on the instrument where the spots from the Earth's surface are sensed. Namely, the conversion of the spots from electromagnetic waves into electrical signals able to be recorded on-board the satellite or transmitted to the receiving stations is done by only one sensor. Furthermore, in this system the response of the sensor has to be fast (short time response) because its output has to fluctuate as the brightness of the spot changes (Lillesand et al., 2004).

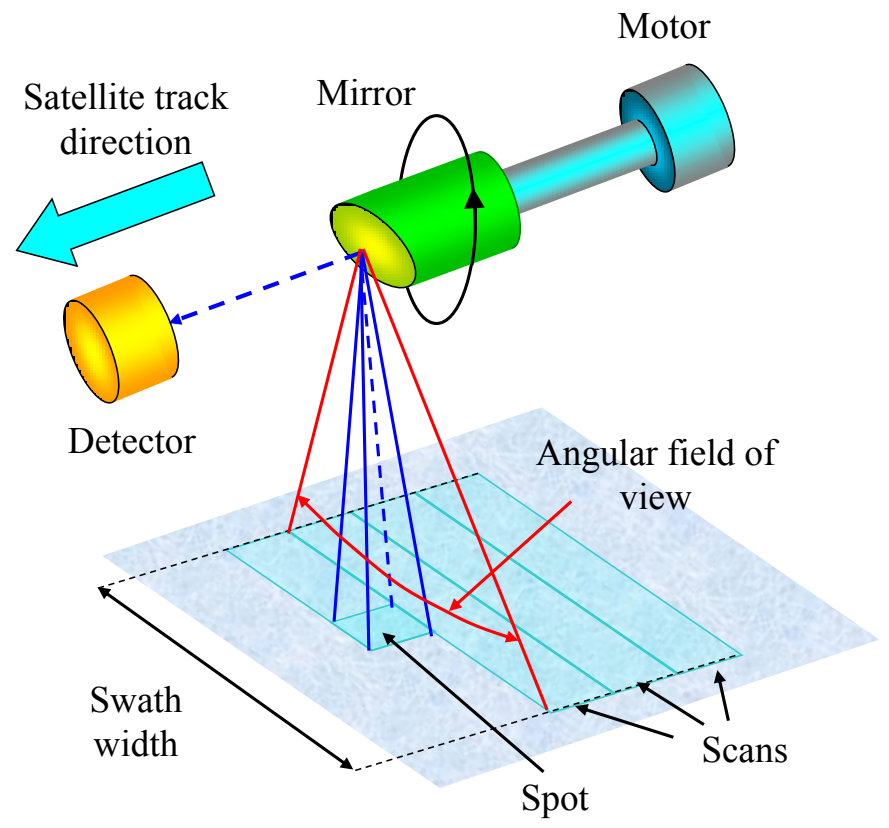

Fig. 10.3: Across-track scanning 
Although there is only one point (called detector in Fig. (10.3)) where the successive spots are converted from optical into electrical signals, multiple sensors or spectral filters can be placed at this point to obtain the same surface's spot information with different spectral resolutions.

The outputs of the sensors are analog functions of time, which are sampled at fixed intervals and converted into digital signals (Section 3.6.4). Digitalization has to be synchronized with the scanning to assign a digital value corresponding to a specific spot on the Earth's surface. Due to variations in the scan mirror velocity some geometric errors are introduced in the sensing process and some pixels could be incorrectly positioned on the image. The main advantage of this arrangement is that all the spots composing the images are weighted by only one calibration constant because the sensor is unique.

\subsubsection{Along-Track Scanning}

In this case the optical system is simpler than in the previous case because many points in a complete line across the track are sensed simultaneously and no rotating mirror is needed. The sensing system consists of a linear array of sensors positioned side by side; the array can include thousands of sensors (Fig. 10.4).

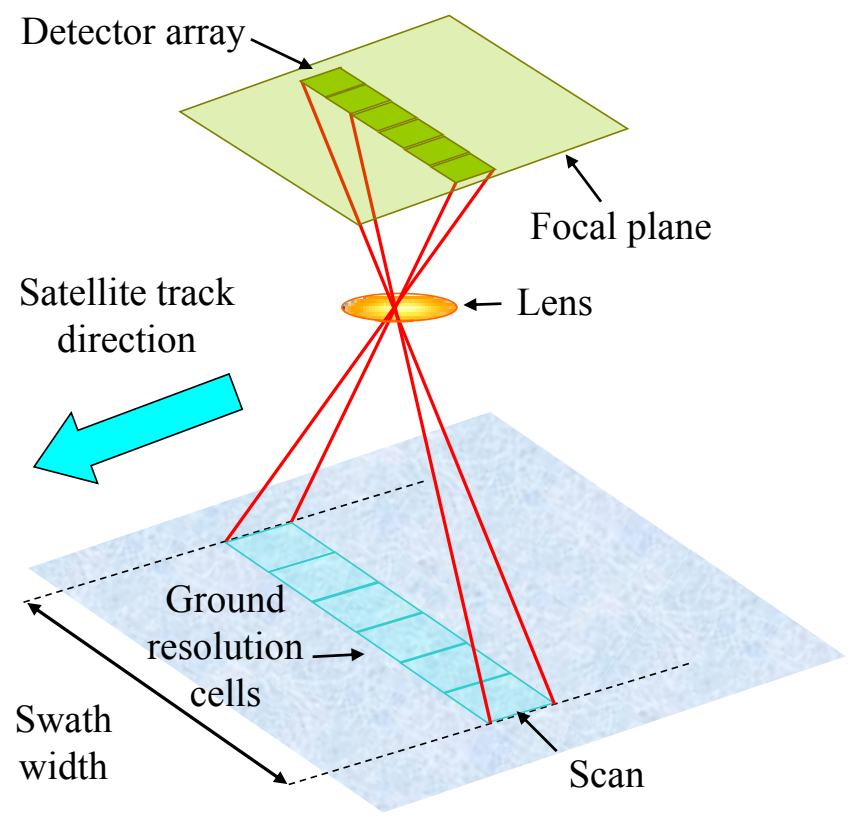

Fig. 10.4: Along-track scanning 
The forward motion of the satellite generates a bi-dimensional image by integrating successive across lines. In this array, the dwell time of an optical input over a sensor can be increased with respect to the across-track scanning because the sensing process is divided in multiple sensors. This enables stronger signals to be recorded, thus higher signal to noise ratios are achieved. Due to the fixed relationship between the sensors of the array, geometric errors are minimized. Also, this array has longer lifetime expectancy because they have no moving parts (Lillesand et al., 2004). A disadvantage of the along-tracking is that to provide reliable images, sensors should be individually calibrated to have their calibration matched. Differences in calibration lead to a lack of homogeneity in the pixels of the same line.

\subsubsection{Rotating Antenna}

Some active instruments use a rotating parabolic antenna as was shown in Figure 8.4. In remote sensing from satellites the antenna are installed on the spacecraft looking down. The antenna is pointed in such a way that the beam describes a cone of vertical axis. It is an inverted version of the system shown in Figure 8.15 where the LIDAR to profile the atmosphere was described.

The antenna is mounted on a spin mechanism that makes the antenna rotate at several revolutions per minute. The spin mechanism provides very accurate spin control, which, in turn, assures an accurate position or the pointing direction, allowing a correct positioning of the spot on the image. Optical encoders such as those described in Section (4.14.2) are used to resolve the antenna direction within a small fraction of degree.

\subsubsection{Digital Video Camera}

The digital camera is conceptually similar to those used for home photography; it senses a certain number of complete lines across the track simultaneously. It is similar to the concept described in Section (4.7.7) for infrared thermography. An image is generated by a matrix of sensors, the number of sensors being the number of pixels in the image.

Multiple-spectral sensors can also be used to acquire information not only in the visible spectra but in other spectral bands as well. Some of these cameras provide images similar to the infrared film (used in aircraft remote sensing) by acquiring three spectral channels, namely in the green, red and infrared. 


\subsection{Passive Instruments}

These measure the Earth's surface radiance, from which meteorological quantities are then derived; other information such as amount of vegetation and volcanic activity can also be acquired. Images are two-dimensional distributions of brightness composed of individual points (pixels), each one with a particular intensity (World Meteorological Organization, 2008). Some parameters that influence the sun reflected radiation are: sun elevation, satellite-sun azimuth angle, satellite viewing angle, transparency of the object, reflectivity of the underlying surface and overlying layers such as thin clouds or aerosols (World Meteorological Organization, 2008).

Water, carbon dioxide and ozone in the atmosphere produce absorption in the infrared bands. Thus, studying absorption in these bands permits the atmospheric temperature and amount of absorbers to be known. Fortunately, there are two regions of the thermal spectrum where atmospheric absorption is low; they are in the wavelength regions from 3 to $5 \mu \mathrm{m}$ and from 8 to $14 \mu \mathrm{m}$ (Lillesand et al., 2004). These atmospheric "windows" allow measuring the energy coming from the surface of the Earth or from the top of the clouds.

In the past, remote images of passive instruments taken from aircrafts were recorded on films. At present, opto-electronic devices are used for recording Earth' surface images. The optical part of these devices is similar to that used in aerial photographic cameras. The electronic components consist of sensors that convert electromagnetic waves to a voltage or a current signal (as it was shown in Sections (4.7.6), (4.7.7) and (7.4)). In the next step of the on-board process the analog signal is converted to a digital one and transmitted or stored (Chapter 9).

\subsubsection{Calibration}

Calibration of the visible channels is performed before a satellite's launch, neglecting the atmospheric effects. This procedure has the disadvantage that the satellite orbital circumstances can be quite different from the calibration conditions. When using data from the satellite it has to be taken into account the radiometer's degradation with time, furthermore, images from different satellites can have different calibration coefficients. For these reasons it would be interesting to develop on-board calibration techniques for the visible channels (World Meteorological Organization, 2008).

Conversely, thermal scanners in the infra red range, which have operating principles similar to that shown in Sections (4.7.6) and (4.7.7), are continuously calibrated on board the satellite. They have two internal temperature references that are acquired together with each scan; thus, a calibration coefficient may be derived for each image's line. These two calibration points are set at both extremes of the expected temperature range to be measured. Additionally, an absolute cold reference 
can be obtained by pointing the instrument to the space, which can be considered as a black body at about $3 \mathrm{~K}$ (World Meteorological Organization, 2008).

\subsubsection{Accuracy}

A common method for evaluating the accuracy of satellite measurements is to analyze the differences between satellite derived parameters and radiosonde profiles. Also, satellite estimations are compared to field data collected on meteorological stations on the land, or on the ocean by means of buoys or ships. Due to the great differences between the temporal and spatial resolutions of the satellite acquired data and those utilized to contrast them, it is quite difficult to assess the accuracy of the satellite measurements. For example, a work aimed to evaluate the quality of the Tropical Rainfall Measuring Mission (TRMM) found differences of concurrent satellite and rain gauge rain rates at various time scales. Correlations for 5, 30, 60 and 1440 minutes were $0.6,0.8,0.85$ and 0.93 respectively. The authors said that the major obstacle in evaluating data quality is the lack of "ground truth" reference at the radar pixel scale. A tipping-bucket rain gauge measures rainwater impinging on a circular surface of few decimeters in diameter. In contrast, radar measures reflectivity factors over an atmosphere column with a base on the order of 1 to $10 \mathrm{~km}^{2}$, and then it estimates rainfall using reflectivity - rain rate relationships. The authors conclude that gauge measurements cannot be directly treated as the ground truth reference due to the lack of areal representativeness (Wang \& Wolff, 2009).

\subsubsection{Passive Microwave Sensing}

Instruments used for passive microwave sensing are called microwave radiometers because they essentially work with the same operating principle as thermal radiometers, but in the microwave range. Because they are passive instruments they sense naturally available energy in the microwave range (wavelength between $1 \mathrm{~mm}$ and $1 \mathrm{~m}$ ) using antennas as sensors (these antennas are similar to those used by active radar).

The information gathered by these instruments is more difficult to interpret than with other instruments because the amount of energy received by the antenna is low and may proceed from several different sources. For example, it may have components due to surface temperature and material characteristics of the object, emission from the atmosphere, reflection of the sunlight and transmission from the subsurface (Lillesand et al., 2004).

Instruments for microwave sensing are classified as radiometers and scanners. The first have an antenna that generates a profile of the energy received but not 
an image. Instead, the second have the ability to move the antenna or use several antennas scanning different swaths; from this information an image is derived.

The interpretation of passive microwave information is not yet well developed, but some interesting characteristics of this technology have emerged. It can be used during day and night under most weather conditions, it is sensitive to water vapor and oxygen, it can be used for identifying sea ice, it can detect soil temperature and oil spills. It has been found that the analysis of passive multi spectral signals in the microwave range gives information from objects beneath the soil; hence it has obvious applications in geology (Lillesand et al., 2004).

\subsubsection{Cloud Drift Winds}

An indirect way for estimating winds from satellites is by evaluating cloud tops' evolution in time. This is carried out by means of infrared images taken from geostationary satellites every half hour. The accuracy of the method relies on the assumption that the cloud motion is due to the local wind, which is not always true because clouds may move due to mesoscale atmospheric disturbances. Another factor that limits the accuracy is the time interval between images.

The process of estimating the wind begins with the selection of target clouds of a size about $20 \times 20$ pixels. This selection is based in the brightness of the clouds. Once the targets are chosen, their motion is evaluated from one image to the next. Results are presented as a wind vector field of about 1200 vectors covering both hemispheres (World Meteorological Organization, 2008).

When the results of this method are compared with wind radar measurements there appear differences of 3, 5 and $7 \mathrm{~m} / \mathrm{s}$ on the mean vector for low, middle and high clouds respectively. This method is not usable in areas without clouds, nor can it be applied for high latitudes because geostationary satellites do not reach high latitudes. In high latitude areas, sequential images from polar-orbiting satellites can be used (World Meteorological Organization, 2008).

\subsubsection{Precipitation}

Some relationships between the brightness of clouds in the visible / infrared spectra and precipitation have been developed such that from images taken every half hour, the precipitation can be inferred. However, these techniques are valid only for certain regions and seasons and they cannot yet be extended in time, or applied to other areas.

One of the methods is called the cloud indexing method. It is based on the definition of some relations between precipitation and the amount and structure of clouds. It consists in evaluating the amount of pixels over the cloud surface, which 
has brightness above a certain threshold. This procedure can be performed with information from the infrared and the visible spectrums. The result of this evaluation is a number called precipitation index.

Some empirical coefficients are adjusted to match the results from satellites to the observations from rain gauges or radar data. Comparisons with rain gauges are difficult because they integrate the precipitation over time in a particular place, while satellite images are instantaneous observations integrated in space.

The cloud indexing method can produce erroneous results due to the presence of non-precipitation clouds, such as cirrus. It has been proposed that satellite results should be used as a complementary tool to ground-based radar observations. Some authors declare that it is easy to adjust coefficients for a particular case, but is difficult to obtain a method that works for most of the cases (World Meteorological Organization, 2008).

\subsubsection{Sea Surface Temperature}

Estimates of ocean surface temperature may be derived from the measurement of radiation emitted by the top surface layer of the ocean (about $1 \mathrm{~mm}$ thick). These estimates are mostly made in the infrared spectrum, and to a lesser extent, in the microwave spectrum. A great advantage is that they are available in real time for meteorological applications.

The measuring process requires removing any pixel covered by clouds and correcting brightness for water vapor attenuation. When the infrared band is used the spatial resolution of the method ranges from 1 to $5 \mathrm{~km}$ (World Meteorological Organization, 2008). Measurements in the microwave band suffer much smaller cloud attenuation and they are an alternative when infrared measurements are not available. The disadvantage of microwaves is that the spatial resolution of these radiometers is of several kilometers.

When the infrared band is used (from 10.5 to $12.5 \mu \mathrm{m}$ ) one way for correcting water vapor attenuation is by taking measurements of the same places from two different look angles because atmospheric attenuation is proportional to the path length. Another way is measuring the same area in two different infrared channels. There are some linear relations between attenuation and wavelength that allow discounting the atmospheric attenuation.

The temperature of the ocean surface measured by radiometers can differ from temperature at a depth of few meters. Some of the factors with direct impact on this difference are: the wind that mixes the surface layers, the sunlight heating the ocean and the rainfall. To reduce these discrepancies daytime and nighttime algorithms are used. In general, nighttime measurements are less disturbed by unwanted conditions.

Measurements from buoys and ships are made at a given point, the depth being about $1 \mathrm{~m}$ to $10 \mathrm{~m}$, respectively. Instead, radiometric measurements are made 
averaging over a large surface layer. In spite of these differences, satellite derived seasurface temperature compares very well with drifting buoys and ship observations; they show differences lesser than $1 \mathrm{~K}$ on average (World Meteorological Organization, 2008).

\subsubsection{Other Parameters Estimated From Satellites}

There are many parameters estimated from instruments on board satellites, and fast advances are going on to refine some measurements and to obtain new ones. For example, the radiance measured at the $6.7 \mu \mathrm{m}$ wavelength is used to estimate the amount of tropospheric humidity in a deep column (not very accurately defined); for this application the segments of the troposphere analyzed should not contain medium and high clouds.

Also, the total ozone density on a column may be measured with radiometers in the ultraviolet band. Some methods combine the information gathered in six wavelengths between 0.28 and $0.3125 \mu \mathrm{m}$ giving ozone measurements within about $2 \%$ of ground based instruments. With a lesser accuracy total ozone can be derived from its absorption in the thermal infrared $(9.7 \mu \mathrm{m})$. Volcanic ash clouds, fires, and vegetation indices are also obtained from the satellite images.

\subsection{Active Instruments}

\subsubsection{General Approach to Active Sensing}

The main advantage of this kind of instrument is that the electromagnetic sources that illuminate the Earth's surface are selected by the instrument designer. The designer can choose the frequency and time characteristics of the wave that will be used to sense the Earth's surface. The benefit of this degree of freedom is that since the instrument knows the characteristics of the signal it is expecting back, it can reject all other signals that do not match the expected pattern. This is done by correlating the received signal with a replica of the sent signal. This selection improves dramatically the signal-to-noise relation.

Also, since the "sensing" signal is produced by the instrument on-board the satellite, the precise instant at which it was emitted is known. Therefore, by computing the time elapsed between the instant in which the signal was emitted and the instant in which the correlation is a maximum, the flying time of the signal between the emitter and the target is calculated. The flying time contains information about the distance between the spacecraft and the target. Furthermore, because the satellite is moving, the signal received back will be of a different frequency than the signal sent. The change in frequency will depend on the speed of the satellite and on 
the position of the satellite and the target (remember the example of the train horn and the concepts introduced in Section (3.3.1)). This information is also used by the measuring systems as will be seen below.

Since sun illumination is not required to get an image, active instruments can be used during night hours. This means that radar images can be acquired on both passes of the satellite, from south to north and from north to south. The radiant sources used by active instruments are generally designed to have frequencies between 0.3 and $300 \mathrm{GHz}$, or wavelengths between $1 \mathrm{~m}$ and $1 \mathrm{~mm}$. Most of these frequencies break through clouds and rain and some targets are "seen" better with them than when illuminated with visible light (GlobeSAR Program, Canada Centre for Remote Sensing).

The microwave spectrum is classified in bands identified by letters; Table 10.1 shows those most often used.

Table 10.1: Microwave bands

\begin{tabular}{lll}
\hline Band name & Frequency range $(\mathrm{GHz})$ & Wavelength $(\mathrm{cm})$ \\
\hline $\mathrm{Ka}$ & $26.5-40$ & $1.13-0.75$ \\
$\mathrm{Ku}$ & $12-18$ & $2.4-1.66$ \\
$\mathrm{X}$ & $8-12.5$ & $3.75-2.4$ \\
$\mathrm{C}$ & $4-8$ & $7.5-3.75$ \\
$\mathrm{~S}$ & $2-4$ & $15-7.5$ \\
$\mathrm{~L}$ & $1-2$ & $30-15$ \\
$\mathrm{P}$ & $0.3-1$ & $100-30$ \\
\hline
\end{tabular}

It is convenient that radar wavelengths are similar to the sizes of the targets from which information is desired (remember the Bragg law (Section (3.5.1)). The $\mathrm{C}$ band is a good choice for most targets. The $\mathrm{L}$ band is the best for geology. Some instruments on board satellites use more than one frequency band (GlobeSAR Program, Canada Centre for Remote Sensing).

Microwaves are electromagnetic waves formed by the oscillations of electric (E) and magnetic (B) fields that are perpendicular to each other and to the direction of wave propagation (transverse waves) (Section 3.4). The oscillations of the electric (E) and magnetic (B) fields lie thus entirely in the plane of the wave front. The most common mode of oscillation of the vector $\mathbf{E}$ (or $\mathbf{B}$ ) is the elliptic mode, in which the linear and circular modes are but particular cases. To see this, suppose that two mutually orthogonal sinusoidal electromagnetic waves of the same frequency and different amplitudes are composed. In general, if the waves are not in phase, 
the tip of the resultant electric $\mathbf{E}$ (or magnetic B) vector travels around an ellipse, and the resultant wave is elliptically polarized. If the phase difference is zero or any integral multiple of $\pi$ the ellipse becomes a straight line, and the resultant wave is linearly polarized. Finally, if the amplitudes are equal and the phase difference is any odd integral multiple of $\pi / 2$, the ellipse becomes a circle, and the resultant wave is circularly polarized. By the way, it is worth noting that in the case of light, which is also an electromagnetic wave, it has been shown, both experimentally and theoretically, that the electric vector $\mathbf{E}$ should be identified as the luminous or optic vector of the electromagnetic wave (Jenkins \& White, 1957; Rossi, 1957).

The designers of the satellites' instruments can select the polarization of the electromagnetic wave transmitted, which is another tool to extract information about the target. The radar signal transmitted from the satellite can present different modes of polarization depending mainly on the antenna system. In general, they are linearly polarized and have polarization planes made horizontal (H) or vertical (V). When the transmitted and received waves are in the same polarization plane they are like-polarized, for example $\mathrm{HH}$ means horizontal transmitted and received. When the transmitted and received signals are in orthogonal planes they are cross-polarized, for example HV for horizontal transmission and vertical reception.

It has been observed that some targets produce different backscatters depending on the wave polarization. The way in which radar waves interact with surface features depends on their slope orientation, soil moisture, vegetation, roughness, etc. Therefore, there may be conditions where the HV images have more information than the $\mathrm{HH}$ ones. For this reason more information is obtained using waves with both polarizations to illuminate the target, thus resulting more detailed images.

Most of the previous chapters deal with instruments such as current meters, wave meters, anemometers, rain gauges, etc that are designed to measure specific parameters. In these cases the names of the instruments have implicit the expected data to be acquired. In active satellite-based remote sensing some instruments are better suited for estimating some particular parameter, but, in general, because they acquire a certain broad spectral band they can estimate several parameters simultaneously. Hence it is not yet simple to name such instruments. Furthermore, the designers of new instruments used to add to the instruments' names some flamboyant words describing the data processing aimed to extract the information from the targets. These characteristics lead to instruments' names that at first sight do not clearly reveal what they measure.

In our opinion, it would be convenient for didactical purposes to separate the concepts on data processing from the instruments' applications. The data-processing concepts are generally used by most of the instruments regardless of the target's parameter to be estimated, so they deserve a separate treatment.

As part of the efforts to synthesize these issues, we will roughly group the on-board data acquisition systems according to three functionalities. One of the functionalities concerns the instruments ability to accurately know the position of the spacecraft at 
every instant of its trajectory. The accurate knowledge of the satellite's orbit to within a few centimeters requires several locating systems on board satellites working cooperatively. Doppler shift techniques are used to determine the satellite's velocity; GPS or similar systems are also used together with laser measurements to know satellite position (Rosmorduc et al., 2011). They are very special instruments giving important information, but this information is generally indirectly used by the environmental science researcher, and for this reason these instruments will not be addressed here.

Another functionality of the acquisition systems is the on-board data processing, which is performed in both analog and digital manners and is implemented by means of electronic circuits or numerical algorithms. They are used to extract information about parameters of interest to the environmental science researcher. These aspects of the problem will be addressed here because they have a direct impact on the final data used by the researchers. We consider it fundamental that data users know how the information has been processed on board the satellite before it arrives at their computers, because this previous process defines the representativeness of the data they will use.

The last functionality to consider is the application of the instruments to estimate some parameters. This subject deals with how the components of the acquisition systems (including the data processing) are disposed to be most adequate to acquire certain specific characteristics of the targets; i.e. it is attempted to describe how instruments are applied and why some of them are more suitable to acquire certain parameters more accurately than others.

Therefore, the first part of the remainder of this chapter deals with data-processing general concepts used in almost all systems, and the second part with a few of the most renamed applications called scatterometers, altimeters and lidars.

\subsubsection{Data Processing On-Board Satellites}

We will describe some operating principles associated with systems such as Synthetic Aperture Radar (SAR), deramp technique, Doppler frequency shift, frequency modulation and Chirp radar. As stated before, this nomenclature is a little confusing because one could infer that SAR and Chirp radar are specific instruments developed to measure a particular parameter, but they are instruments of general applications. In reality one might think that they are general concepts on data processing.

All active instruments are devoted to extract information from the backscatters received at the satellite antenna. Thus, they are simply emitters of electromagnetic waves towards the Earth and collectors and processors of the backscatters coming from the Earth. The differences between these instruments are in the frequency they transmit, the kind and number of antennas, the on-board electronics and the way they process the data. But, in general, even when an instrument is designed to be better suited to perform a specific measuring task, it will provide much more information than that for which it was tuned. 


\subsubsection{Synthetic Aperture Radar (SAR)}

SAR does not name a specific instrument but a group of radar systems that use a particular way of acquiring and processing the received backscatters. One feature that differentiates this measuring method is that it is used in moving active instruments (which emit a well-known wave) and whose spatial positions are well known at any instant. The main characteristics of the operating principle that distinguish SAR technology will be described using some concepts already introduced in previous chapters; therefore it would be convenient to have them present now.

Section (3.5.2) illustrates an array of sensors located at different spatial positions with the aim of measuring a propagating wave field. It was stated that such an array permits the wave field at different places to be known as a function of time. These data may be combined to enhance the signal-to-noise ratio beyond that of a single sensor's output.

Also, the beamforming concept was introduced in Section (3.5.3). It was also stated that processing signals from several antennas arranged in a certain spatial allocation permits the signals to be processed to reinforce some signal coming from a particular direction, rejecting others.

Figure 3.8 shows a linear array of transducers receiving the signal from a scattering source. A similar case is now presented in Figure 10.5, where the transducers are replaced by receiving antennas and the scattering source is a particle that was irradiated by an electromagnetic pulse coming from a transmitting antenna. Since the distances from the source to each receiving antenna (d1, d2, d3 and d4) are not the same, the scattered signals will follow different paths, suffering different delays to arrive at the receiving antennas and showing a different degree of attenuation.

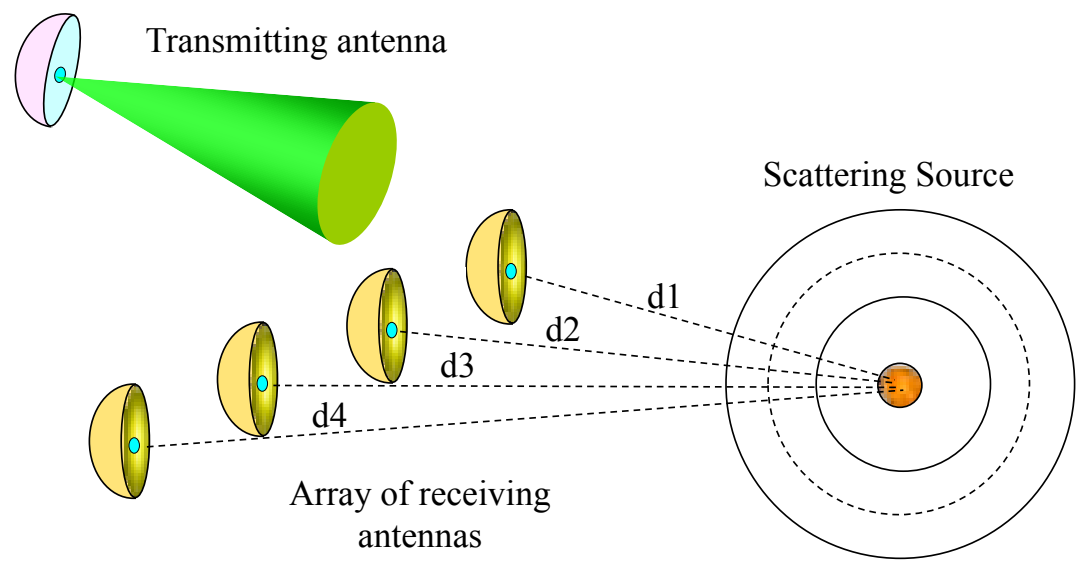

Fig. 10.5: An array of antennas receiving electromagnetic energy scattered by a particle (source). The distances between the particle and the antennas are $\mathrm{d} 1, \mathrm{~d} 2, \mathrm{~d} 3$ and $\mathrm{d} 4$. 
Recall now Section (8.10.1) where it was shown an array of receiving antennas fixed on a beach and a couple of transmitting antennas that irradiate the sea with electromagnetic pulses. In this case all the receiving antennas simultaneously acquire the signals coming from the sea (scattered by the waves). The signal received at each antenna can be processed (basically phase shifted) and the resulting signals added. Thus, only signals coming from a given direction will add in phase (beamforming). This array of antennas and the data processing permit focusing on some small area of the scene. The signals received over the antenna array can be recorded and reprocessed differently several times (De Chiara et al., 2007). Thus the results for each processing step can be combined and displayed together.

There is a relation between the spatial resolution, the length of the antennas' array $(A)$ and the transmitted wavelength $(\lambda)$ (Campbell, 2007):

$$
\beta=\frac{\lambda}{A}
$$

where $\beta$ is the beamwidth of the array of antennas. A narrow beamwidth means a higher spatial resolution and according to Eq. (10.1) the longer the antenna array (A) the better the spatial resolution. For this reason several antennas were equally spaced on the beach. As explained in Sections (2.3.2) and (2.3.3), the beamwidth defines, in part, the spatial resolution. The other factors defining the spatial resolution of a radar system are its frequency and the pulse width (Section (8.2)).

Consider again the antennas fixed on a beach. If the scattering of the target under study would change very slowly with time, successively placing the same antenna at the position of each one of the antennas of the array would sequentially record "similar" information as all of them would also do. In other words, by displacing only one antenna, "similar" information as that simultaneously recorded by the whole array could be obtained. Later on all this information can be processed (delayed and summed up) to enhance the signal-to-noise ratio, which improves the information quality, since it is usually done for the fix complete array.

Suppose now that we have one transmitting $(T x)$ antenna and one receiving $(R x)$ antenna (Fig. 10.6) and that an orbiting satellite carries this pair. The position of the antennas as a function of time is known due to the on-board instruments to accurately position the spacecraft. The target scene is repeatedly irradiated by electromagnetic pulses sent from the transmitting antenna. The scattered signals are received back by the receiving antenna at its successive positions and stored.

In the example shown in Figure 10.6, signals from four different positions are stored in a similar way as the four fixed antennas in Figure 10.5. Later, the stored signals are read out and combined using specific phase shifts. The result is equivalent as if the signals were recorded simultaneously by an array of equally spaced antennas fixed at four points of the satellite orbit. 


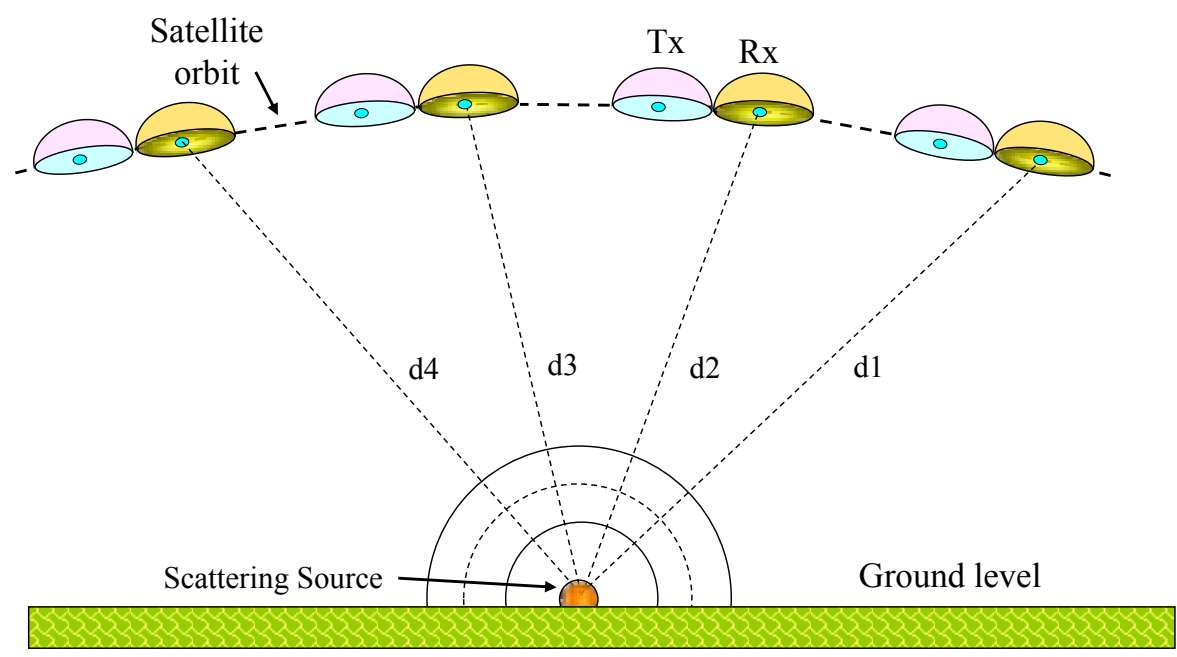

Fig. 10.6: The scattering source is irradiated by the transmitting antenna from four different positions. The scattered signals are received back by the receiving antenna at its successive positions.

In the example, two antennas, one for transmitting and other for receiving were used only with didactic purposes. As it was mentioned in Section (8.2), only one antenna can be used to transmit and receive; a device called duplexer can switch the antenna between the transmitter and receiver. Only one antenna is used by the SAR. This is the working principle of a Synthetic Aperture Radar (SAR); a moving antenna can gather "similar" information as an array of fixed antennas can do.

Recapitulating, radar on board a satellite moving at constant speed and acquiring information from different positions performs like an array of antennas. Remember that the array provided with an intelligent data processing (beamforming) performs as a narrow beamwidth, which allows focusing on some small area of the scene giving a higher spatial resolution. Therefore, SAR is synonymous to high spatial resolution due to a specific data processing.

Going a little deeper with the data processing and keeping in mind the Doppler effect, the reader can infer from the concepts developed in Section (3.3.1) that the backscatter's frequency is different than the sent frequency. This is so because, albeit the targets are fixed, the radar mounted on the satellite is moving. At a given instant, a target illuminated by the leading edge of the radar beam will reflect a frequency higher than the transmitted one (see Section (3.3.1)). Instead, when the target is in the trailing edge of the beam the reflected signal experiences a decrease in frequency. Because the radar system is able to calculate these frequency shifts, it is possible to use them to accurately know the relative position of the backscatters at each instant. 
Thus, due to the frequency shifts, when composing and image using the information provided by the backscatters it is possible to assign each scatter to its correct positions on the image.

We want to stress once more that the acronym SAR does not describe an instrument for measuring a particular parameter, but several instruments with different names can be said to be SAR systems (e.g. scatterometers, altimeters, etc).

\subsubsection{Chirp Radar}

This is another data processing method to improve signal-to-noise ratio. It is also known as linear Frequency Modulation (FM) pulse compression. The name chirp refers to 'chirping', i.e. to make short, sharp notes or sounds (as small birds or insects), and it is also the acronym for "Compressed High Intensity Radar Pulse".

In contrast to old radars, which relied on the transmission of short-duration continuous wave pulses with a peak power of several kilowatts, most modern instruments transmit linear frequency-modulated pulses of longer duration and a relatively lower peak power. These frequency-modulated pulses are also known as "chirps". This concept was initially used in military applications and the simplest way to explain how chirp radars work is by following one of the first public documents on this subject (Klauder et al., 1960).

Suppose that the transmitting signal of a radar, instead of being one short pulse of a single frequency, is comprised of a sequence of adjacent pulses each one of a particular frequency $\left(f_{N}\right)$; frequencies are arranged in increasing order as in Figure 10.7a, and this resulting signal is transmitted to illuminate a target.

Assume that in the receiver, there is a network possessing delay versus frequency characteristic as in Figure 10.7b, and that it is introduced in series in the backscatter signal path so that the signal will be delayed in steps. Then the signal with frequency f1, which was the first emitted, will be the first received, but will be the most delayed. Each successive pulse of a different frequency is delayed in Td until the last signal arrives to the receiver. Then, all pulses are made to emerge together from the delay network. It may be thought as though the signals are summed up in phase. In this way, the original subsequent pulses of Figure 10.7a are compressed in time and increased in amplitude.

In the previous example, the frequency is changed in steps. In real chirp radar the signal transmitted has also constant amplitude but it is linearly modulated in frequency (Fig. 10.8a). It is centered at frequency fo and has a bandwidth $\Delta$ f. In this case the delay network is a linear function of frequency (Fig. 10.8b). Thus, the backscattered signal at the network output will also produce a compression in time and an increase in amplitude. 

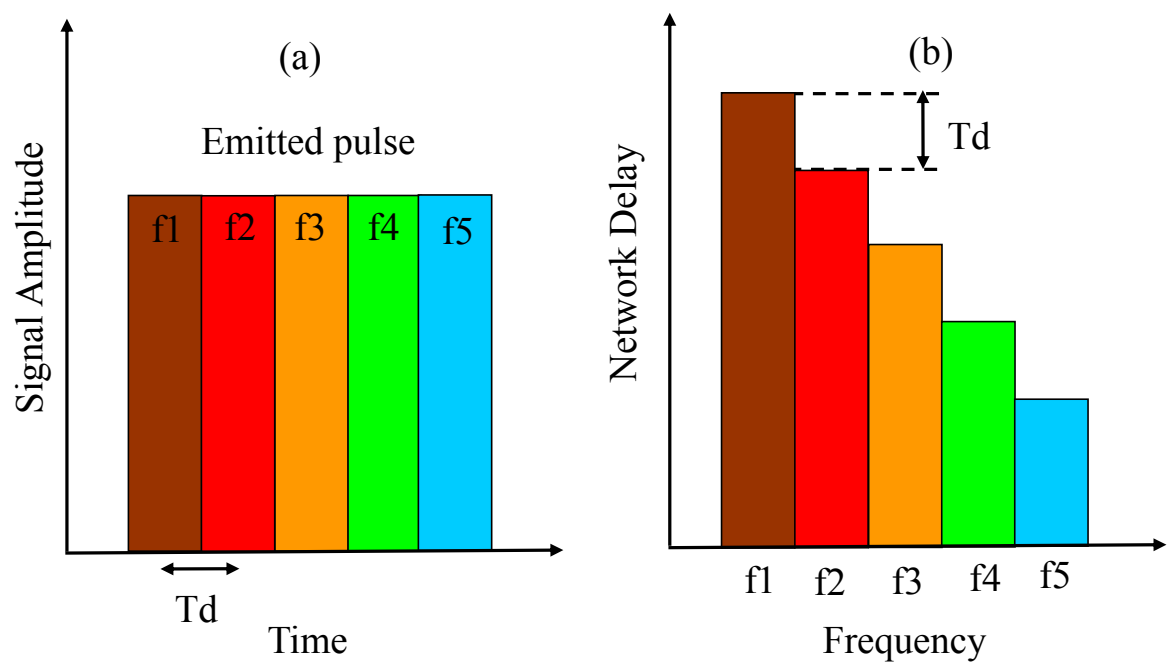

Fig. 10.7: The emitted pulse is an array of consecutive pulses of time length Td and different frequencies. The received signal enters the Network Delay, which retards each frequency a time Td.

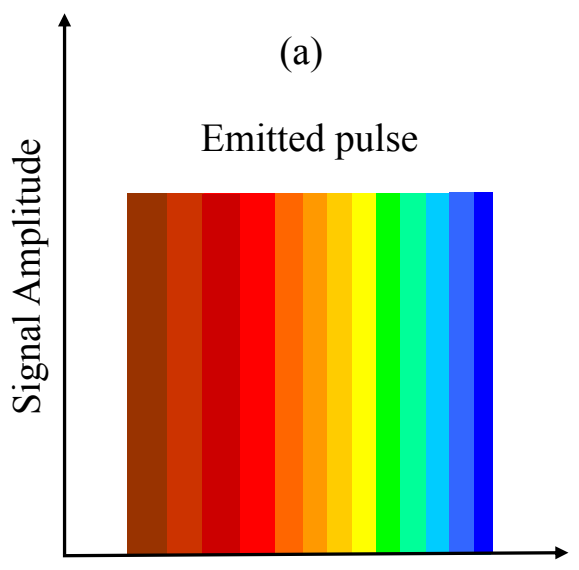

Time

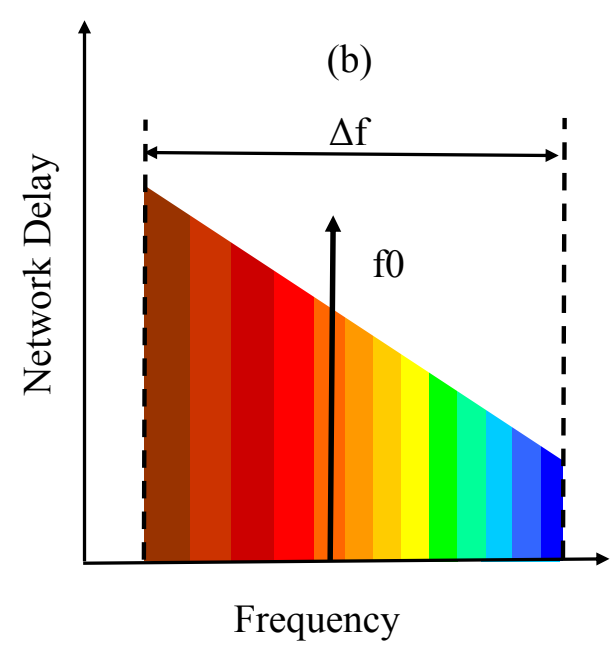

Frequency

Fig. 10.8: The emitted pulse is a signal linearly modulated in frequency whose central frequency is fo and the bandwidth is $\Delta \mathrm{f}$. The Network Delay produces a linear retard as a function of frequency.

This process may be imagined as a number of echo signals of different frequencies arriving in a certain period of time that are superimposed, signal overlaps and the random noise averages, raising the signal-to-noise ratio. 
Hitherto, the signal processing was aimed at rescuing the signal from the noise. Remember that the received signal is an echo from a target whose characteristics we want to know. Hence a new step has to be performed to rescue the flying time of the pulse in both directions that contain the information of the target range (Section (8.2)).

The output pulse of the delaying network is retarded with respect to the transmitted chirp for an unknown time due to the round-trip time of the pulse. Figure 10.9 shows $T x$ as the transmitted chirp, $B$ as its bandwidth and $T$ as its length. A replica of the transmitted signal (Reference) is retarded purposely for a known amount $(D)$ called deramp time delay. $D$ is intended to match approximately the arrival time of the received echo which should be equal to the two way travel time from the radar to the target (Cotton et al., 2008).

When the received echo is compared to the Reference, the difference in time between both signals $(t d)$ will be the difference between the real flight time and $D$. As $D$ is known, measuring $t d$ will give the flight time.

Since the emitted pulse was linearly modulated in frequency as a function of time, a difference in time between these two signals will appear as a difference in frequency. Therefore, the time difference can be extracted from the difference in frequency which is related to the original chirp slope by

$$
t d=\frac{\Delta f T}{B}
$$

By mixing the received echo and the reference, differences in frequencies will be made evident (as explained in Section (8.2) for synchronous demodulation). Low pass filtering and spectral analyzing the mixer output allows finding these frequency differences and then $t d$. Hence, the time of flight can be calculated as $D+t d$, and consequently, the target's range (considering light speed constant). This data processing method is called a full-deramp technique (Chan \& Koo, 2008).

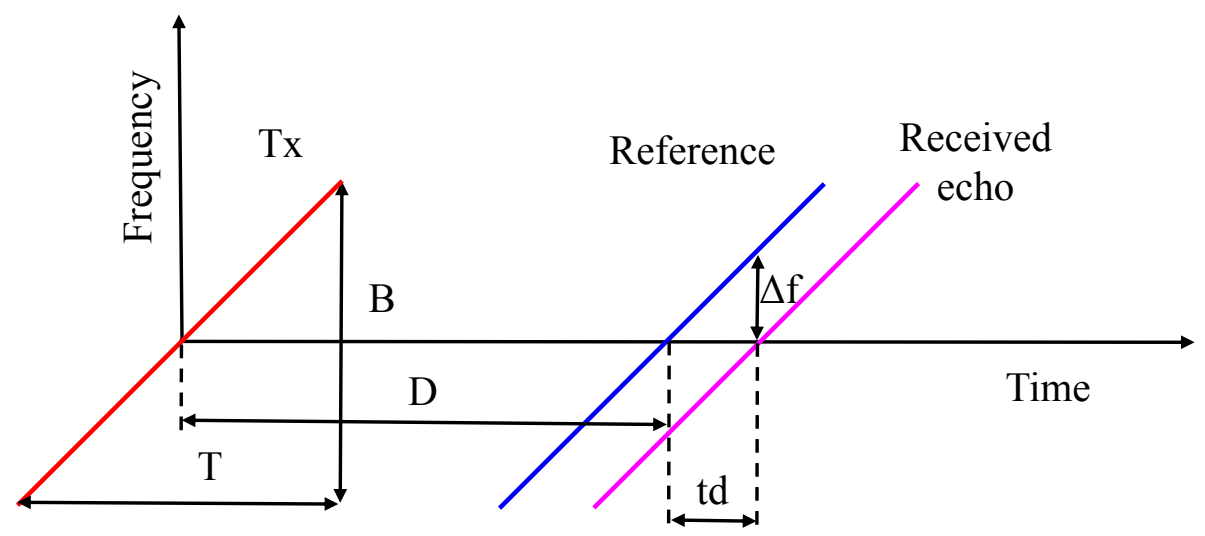

Fig. 10.9: Tx is the transmitted chirp, $B$ is the chirp bandwidth and T the chirp length. Reference is a replica of the transmitted signal delayed a deramp time $D$. The received echo is delayed td with respect to Reference. The time td can be obtained from $\Delta$ f. 


\subsubsection{Examples of Applications of the On-Board Instruments}

On board instruments make use of most of the data processing techniques described above. SAR, chirp radar, Doppler shift, frequency modulation and deramp techniques are used in scatterometers, lidars and altimeters to extract Earth's surface information.

\subsubsection{Scatterometers}

Scatterometers used to measure near-surface ocean winds are basically microwave radar that transmits high frequency electromagnetic waves towards the ocean surface. This energy is scattered by the ocean waves and, as explained in several sections of Chapter 8, the backscattered signal carries information on the target. The data processing operation allows drawing the information of interest from the backscatters received at the satellite antenna.

In addition to the data processing there are other design features that define the ability of the radar to extract particular information from the target. For example, it has been shown in Section (8.9) that the maximum of the scattered energy depends on the relation between the surface roughness of the sea and the illuminating wavelength of the radar (Bragg law). It should also be considered that some high electromagnetic frequencies are strongly attenuated by the clouds, thus being useless to measure surface ocean winds during cloudy days. Therefore, the selection of the scatterometer frequency determines the kind of information that can be gathered from the Earth's surface.

The number, direction and polarization of the antennas which receive the backscattered signals define the quality and the redundancy of the information collected. More "points of view" or "viewing angles" means more signals to be processed and more possibilities of getting detailed pictures of the target. The beamwidth of the antennas and the satellite's orbit altitude play an important role in defining the spatial resolution of the radar "images".

\subsection{Wind Measurements}

The concept behind the measuring method of sea surface winds from satellites with a scatterometer is that the roughness of the sea surface depends on the wind speed. It is a daily experience that a flat lake surface exists when there is no wind; small ripples appear with moderate winds, and a rough surface is observed under high-wind conditions. With no border conditions limiting wave propagation, wind direction and wave front direction have a defined relation.

Electromagnetic waves from radar are used to evaluate the sea surface roughness; although they do not penetrate sea water, the backscatters on the sea surface are completely influenced by surface roughness. Backscatter presents a wide level range of energy which is directly related to wind speed and direction (for a constant 
radar incident angle). Therefore, the fraction of energy returned to the satellite (backscattered by the waves) is a function of wind speed and wind direction (Center for Ocean-Atmospheric Prediction Studies, Florida State University).

Radar pulses in the C-band have wavelengths of about $5 \mathrm{~cm}$ while ocean windgenerated capillary waves and gravity waves are in the order of the radar wavelength; hence Bragg resonant scattering is highly probable. These short-length windinduced waves (mounted on longer waves) modify their amplitudes according to the wind speed, thus changing the roughness of the sea surface. Therefore, the local backscattered energy increases with wind speed. But if the wind produces high sea states, the radar ability to detect sea features decreases due to the higher level of turbulence. When the direction of the wind and the direction of the radar beam agree, the backscatter is the highest. Also, some difference is found between upwind and downwind directions (GlobeSAR Program, Canada Centre for Remote Sensing).

The theoretical function describing the sea surface backscatter coefficient as a function of wind direction, and for a given incidence angle of the scatterometer, is shown in Figure 10.10 (Gelsthorpe et al., 2000). For each wind speed it has two minima, one relative maximum and one absolute maximum. Each wind speeds have a different letter color from A (red - maximum speed) to E (blue - minimum speed). Theoretically the absolute maximum corresponds to the true direction of the wind, the relative maximum to the opposite direction, and the two minima to directions approximately perpendicular to the wind direction.

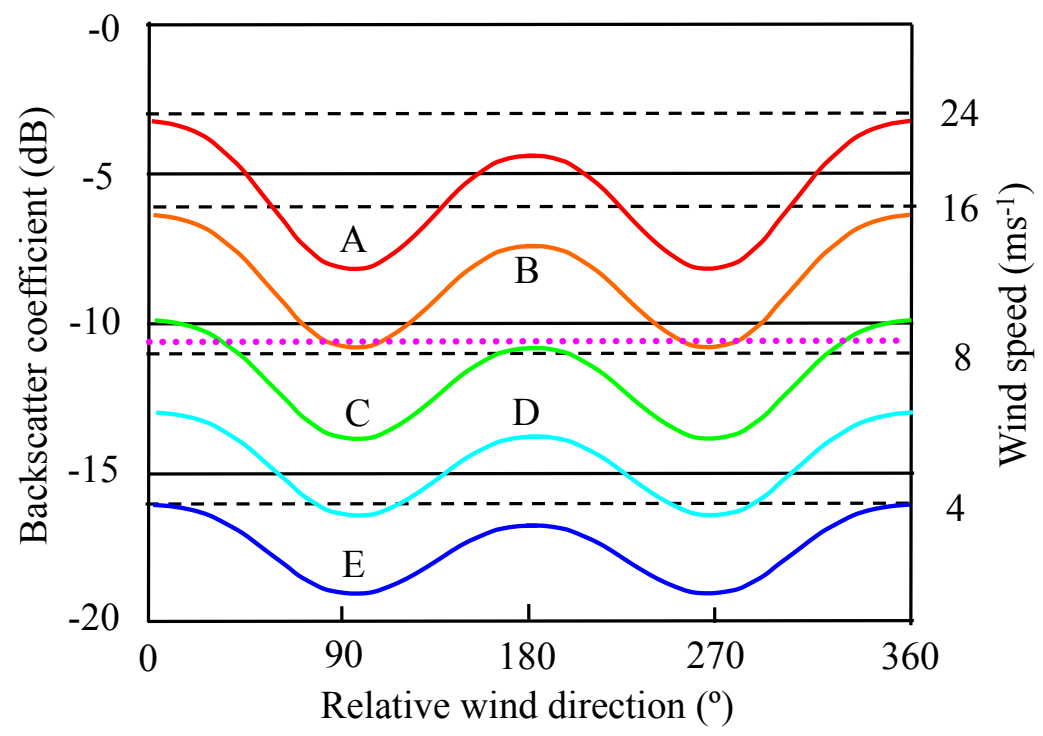

Fig. 10.10: Schematic of the sea surface backscatter coefficient as a function of wind direction for several wind speeds (different letters $A-E$ ) and for a fixed incidence angle. 
As is obvious from Figure 10.10 there exist many possible wind speeds generating the same backscatter; hence, estimating wind speed from only one measured backscatter generates multiple ambiguities. For the dotted line ( $-10.5 \mathrm{~dB}$ coefficient), there are several winds that can generate this backscatter. The process of selecting the direction from the graphic is called ambiguity selection. Problems with ambiguity removal exist for low wind speeds because the signal is weak and easily corrupted by noise. For wind speeds greater than $8 \mathrm{~ms}^{-1}$ successful ambiguity removal is highly probable (Center for Ocean-Atmospheric Prediction Studies, Florida State University).

The method of removing ambiguities relies on "seeing" the same backscatering ocean area from several different relative directions between the beamwidth and the wind. For this purpose modern scatterometers have several antennas with different looking angles, and in some cases they are mounted in a spinning mechanism as described early in Section (10.5.4).

Since 1996 three successive scatterometer missions have been operated and some periods of data overlapping exist; two instruments used C-band frequency and one Ku-band frequency. Bentamy et. al. (2013) were interested in constructing a consistent scatterometer time series despite changes in scatterometer technology. The study focuses on scatterometers that use transmitting frequencies $5.3 \mathrm{GHz}(5 \mathrm{~cm})$ and $13.4 \mathrm{GHz}(2 \mathrm{~cm})$. Because the ocean surface radar signal backscatter occurs from capillary/gravity waves of a few centimeters in amplitude, waves with about $5 \mathrm{~cm}$ and $2 \mathrm{~cm}$ scatter most efficiently at the above frequencies (Bragg scattering).

Due to the shorter wavelength, Ku-band scatterometers are more sensitive to impacts of rain than the longer wavelength C-band; undetected rainfall preferentially affects the higher frequency. Satellite estimations of winds are perturbed due to several different causes: attenuation by raindrops in the atmosphere, amplification due to volume scattering and changes in the sea surface roughness by impinging drops. Some authors have found systematic biases in the $10 \mathrm{~m}$ equivalent satellite wind speed that are a function of radar azimuth angle and wind speed ranges and rainfall, but wind direction does not result affected (Bentamy et al., 2013).

\subsection{Rain Measurements}

In Section (10.6.5) it was anticipated that precipitation can be estimated from passive instruments through relationships between brightness of clouds in the visible / infrared spectra and precipitation, but if it were required to extend rain estimation to night hours, the previous technique is not useful.

The incorporation of data collected by means of active instruments improves precipitation estimation. Some satellite missions devoted to measure rainfall in tropical regions use active sensors called Precipitation Radar (PR). The PR can derive the three-dimensional structure of precipitation. Its operating frequency is $13.8 \mathrm{GHz}$. The PR has a minimum detectable rain rate of $0.5 \mathrm{~mm} / \mathrm{h}$ with range resolution of 
$250 \mathrm{~m}$ (Oki et al., 2010). Combining data from the PR and passive instruments greatly improves rainfall estimation from satellites.

Development of another instrument called dual PR (DPR) is underway; it is composed of two radars, one in Ku-band (13.6-GHz) and other in Ka-band (35.5-GHz). Simultaneous observation of KaPR and KuPR would enable accurate measurement of precipitation from heavy rainfall in the tropics to weak snowfall at high latitudes. $\mathrm{KuPR}$ is suitable for heavy rainfall while KaPR can estimate weaker rainfall and snowfall (Oki et al., 2010).

Cohen Liechti et al. (2012) analyzed rain data gathered from three different satellites (that will be identified here as A, B and C) during four years (2003 - 2007) with the objective of using them in modeling the hydrological behavior of a basin. These investigators found that global correlation coefficients were 0.54 between Satellite A and B data sets, 0.76 between Satellite A and C and 0.60 between Satellite $\mathrm{B}$ and Satellite C. They concluded that although in terms of yearly rainfall the main characteristics are preserved, the rainfall spatial patterns produced by the three satellites show considerable differences, which illustrated the very strong dependency of the satellite product quality on the region studied (Cohen Liechti et al., 2012).

\subsection{Moisture}

In Section (4.9), when describing humidity sensors, we saw that the conductivity of some materials changes with their moisture content. Also, the complex dielectric constant of a material depends on the moisture presence. The dielectric constant for water is about ten times that of dry soil. Because the dielectric constant defines the ability of a material to reflect, transmit or absorb electromagnetic energy, the scattered energy from a given surface changes according to its water content. Therefore, the same area of soil in a radar image will have a different appearance if it is dry or wet. This is the reason why radar images, when calibrated, can be used to infer the moisture content of a certain soil. In wet areas the radar waves do not penetrate the subsurface, but dry areas such deserts are easily penetrated by radar waves. Thus, the scatters have information not only on the surface properties but on the subsurface too.

Radar from satellites can quantify moisture on the surface of soil from 0 to $15 \mathrm{~cm}$ in depth, but the strongest correlation is for the $0-3 \mathrm{~cm}$ depth range. A stepped incident angle gives better results because it minimizes roughness contributions. The moisture content of frozen soil cannot be measured with these methods. Also, rain and dew degrade the acquisition of moisture content in soil.

\subsection{Ice}

Not only can satellite's radar (SR) identify ice, they can also estimate ice type and surface roughness as well. If the ice surface is smooth there is a good reflection and it appears dark. Instead, when roughness increases the area appears bright. This simple 
relation between surface roughness and brightness is spoiled when melting snow covers the ice surface.

First year ice has a large dielectric constant due to its salt content; hence the radar wave does not penetrate it. Multi-year ice that has several melt seasons has lower levels of salinity and hence lower dielectric constant. In this case the radar wave has a greater probability of penetrating the ice and the scatters come from the surface and subsurface layers. This makes it to appear brighter when compared with first year ice. SR cannot measure ice thickness directly, but discriminating ice age permits inferring ice thickness (GlobeSAR Program, Canada Centre for Remote Sensing).

\subsection{Other Applications}

SR can be used for ship detection and oil spills monitoring because surfactants produce a localized suppression of the small waves that give rise to Bragg scattering, but SR cannot estimate oil layer thickness. Specular reflections from water produce dark images. Thus, flooded areas can be distinguished, but wet snow can be confused with flooded areas (GlobeSAR Program, Canada Centre for Remote Sensing).

Since the scattering of a volume of vegetation is stronger when the radar wavelength is similar to the mean size of the plants components, some radar frequencies are more suited for specific crops. For example, wavelength between 2 and $6 \mathrm{~cm}$ are sensible for detecting corn, soybeans, wheat and tree leaves. Instead, for sensing tree trunks and limbs wavelengths between 10 and $30 \mathrm{~cm}$ are more adequate (Lillesand, et al., 2004).

\subsubsection{Altimeters}

This explanation will begin with the operating principle of a simple altimeter, and in a second step the characteristics of more realistic ones will be described.

The altimeter antenna emits pulses toward the nadir hitting the Earth's surface (water or land). Frequencies are in a range from about 3 to $13 \mathrm{GHz}$. The beamwidth is narrow but the wave front widens as it travels downward. When the wave front hits the Earth's surface it has the shape of a spherical cap. If the hit area is plane, the footprint of the leading edge of the pulse is a disc linearly spreading with time until the arrival of the rear edge of the pulse. From then on, the footprint becomes a ring with increasing radius and constant area (Fig. 10.11) (Dinardo, 2013).

The strength of the backscatter (Fig. 10.11d) is proportional to the footprint area, thus the signal retuned to the altimeter antenna will rise linearly up to a maximum corresponding to the passage of the rear edge of the pulse over the Earth's surface. From this moment on, the returned signal should be theoretically constant, but due to practical reasons such as the antenna beamwidth it decays until it arrives at the noise level. 

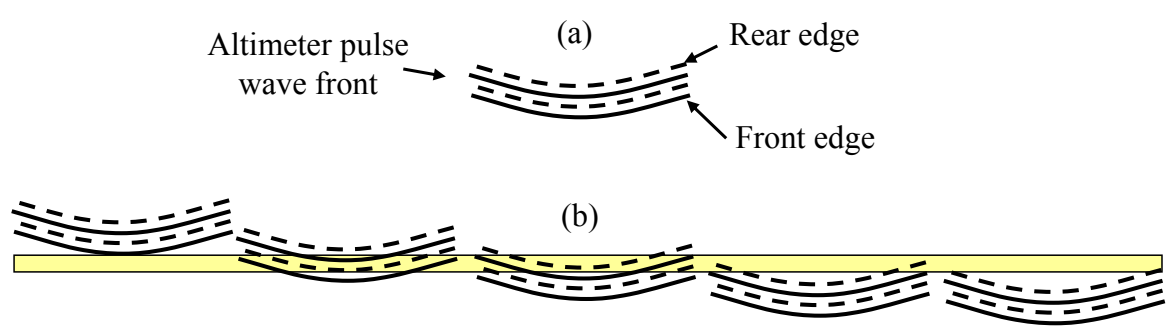

(c)

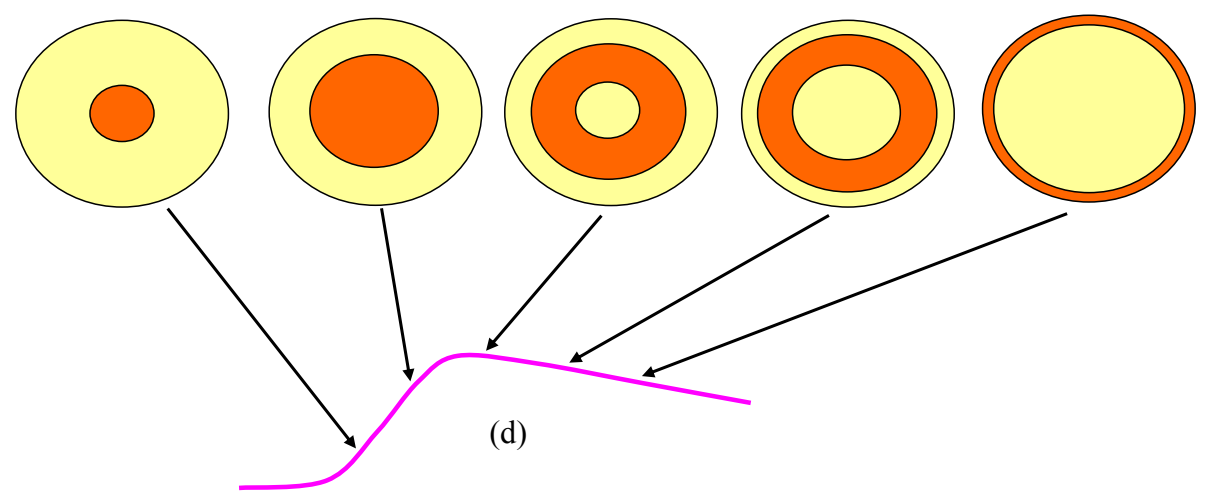

Fig. 10.11: (a) A curved wave front arriving at the Earth's surface. As the pulse hits a plane surface (b) the footprint changes as a function of time (c). The shape of the echo is, up to some point, proportional to the footprint area (d).

The previous explanation was for a plane surface, but if the wave front hits the ocean surface, due to the wave heights, the returned echoes will travel different distances to arrive back at the satellite. This effect produces a change in the footprint, which is a function of the sea state. For this reason, the echo rise time is longer and it is possible to relate the echo slope to the wave height. Therefore, the shape of the signal returned to the altimeter antenna contains information about the wave heights (Rosmorduc et al., 2011).

As could be intuitively perceived, the data are affected by statistical fluctuations because the signals received at the altimeter antenna are composed of many scatters that are not in phase. Therefore, to reduce the noise the instrument averages the echoes.

A more sophisticated altimeter is the delay-Doppler altimeter which, as a first approach, is similar to the instrument just described but with an increased complexity in the data processing method. Since the position of the altimeter as a function of time is known because the position of the spacecraft is known, the same processing methods explained for the Synthetic Aperture Radar (SAR) can be applied. The 
backscatters are recorded at the successive positions of the altimeter and combined using specific phase.

Once again the beamforming concept is used to obtain higher spatial resolution in the along-track direction.

For example, in the CryoSat-2 altimeter 64 beams per burst are synthesized in the along-track direction. As a result, the resolution is sharpened whereas the across-track remains with the previous diameter of the pulse-limited circle. Figure 10.12 shows how the along-track beamforming increase the resolution on the footprint for three of the cases previously shown in Figure 10.11. For the CryoSat-2 case the resolution is $300 \mathrm{~m}$ after the data processing (Dinardo, 2013).

Along-track

synthetic

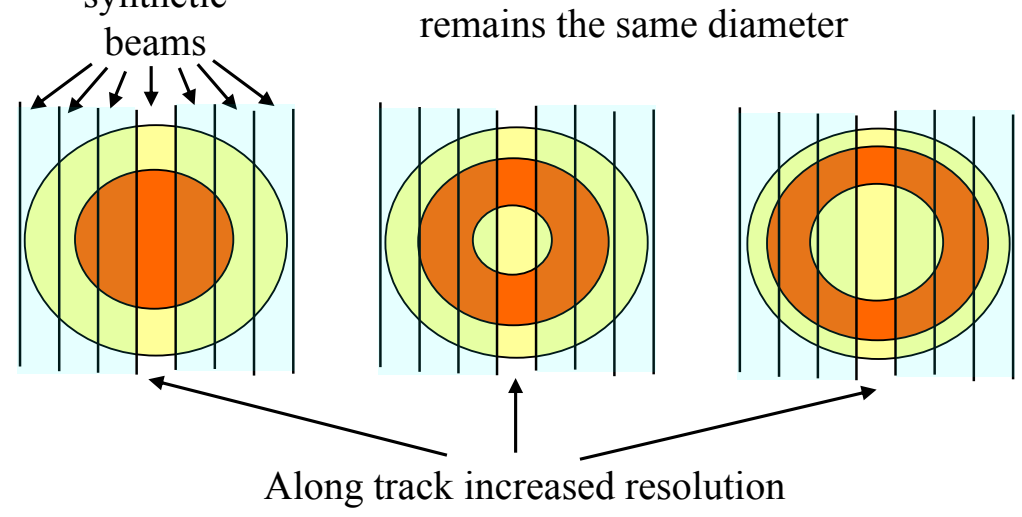

Fig. 10.12: The along-track synthetic beams increase resolution in the satellite track direction, whereas in the cross-track the resolution remains the same.

It was described for the SAR that the Doppler effect due to the satellite movement with respect to the target causes differences between the sent and received signal frequencies. Returned waveforms are frequency analyzed and used to separate the radar footprint into "strips" of increased resolution. Coherent processing (beamforming) of echoes allows for multiple "looking directions" for a given strip, increasing signal to noise relation.

\subsubsection{Lidar}

Lidar operating principle used in satellites is quite similar to that described in Section (8.8). In that case, the laser was pointed towards the atmosphere; in the spacecraft, the instrument is mounted such that the laser pulses are sent towards the ground, thus illuminating a fraction of the surface. The earliest systems were used to profile 
ground's elevations under the spacecraft by measuring the time elapsed when a pulse is sent and when it is received back.

In those cases where the laser beam can pass through the water it can be used to measure the water depth (bathymetry). Each pulse from the laser illuminating the water body produces two echoes, one at the surface and one at the bottom, the second being the weaker. Measuring the time difference between these two echoes permits the water depth to be estimated.

In an urban area or a forest, each lidar pulse can return several echoes due to the landform. The time differences allow estimating intermediate distances, for example, the height of the top of a building, the top of the trees, etc.

In order to know height with accuracy it is necessary to know very accurately the satellite's orbit. For this purpose ground stations with well-determined positions are used to establish the distance to the satellite. Range resolutions in ground elevation from 10 to $30 \mathrm{~cm}$ are common.

The first NASA lidar flying on board a satellite was lunched in early 2003 and worked at 1.064 and $0.532 \mu \mathrm{m}$ wavelengths. It transmitted 40 pulses per second and measured the echo to within $1 \mathrm{~ns}$. The laser footprint was about $70 \mathrm{~m}$ in diameter and they were spaced $170 \mathrm{~m}$ along-track. This lidar can measure small changes in ice sheets and was aimed to study the balance of polar ice sheets and sea level (Lillesand et al., 2004).

\section{References}

Astrium Geolnformation Services (2013) Available at: info@astrium-geo.com/es/www.spotimage.com

Bentamy A., Grodsky, S. A., Chapron, B., \& Carton, J. A. (2013). Compatibility of C- and Ku-band scatterometer winds: ERS-2 and QuikSCAT. Journal of Marine Systems, 117-118, 72-80.

Campbell, J. B. (2007). Introduction to Remote Sensing. New York: The Guilford Press.

Center for Ocean-Atmospheric Prediction Studies (COAPS), Florida State University Available at: http://coaps.fsu.edu/scatterometry/about/overview.php

Chan, Y. K., \& Koo, V. C. (2008). An Introduction to Synthetic Aperture Radar (SAR). Progress in Electromagnetic Research B, 2, 27-60. Available at: http://www.jpier.org/PIERB/ pierb02/03.07110101.pdf

Cohen Liechti, T., Matos, J. P., Boillat, J. L., \& Schleiss, A. J. (2012). Comparison and evaluation of satellite derived precipitation products for hydrological modeling of the Zambezi River Basin. Hydrology and Earth System Sciences, 16, 489-500. Available at: www.hydrol-earth-syst-sci. net/16/489/2012/

Cotton, D., Andersen, O., Cipollini, P., Gommenginger, C., Quartly, G., Martin, C., Marquez, J., \& Moreno, L. (2008). Development of SAR Altimetry Mode Studies and Applications over Ocean, Coastal Zones and Inland Water. SAMOSA. ESA A0/1-5254/06/I-LG, Satellite Observing Systems.

De Chiara G., Crapolicchio, R., \& Lecomte, P. (2007). ERS-1/2 Scatterometer new products: mission reprocessing and data quality improvement. ESA. Earth Observation. Product Control Service. Second Space for Hydrology Workshop, Geneva, Italy. Available at: http://earth.eo.esa.int/pcs/ ers/scatt/articles/Extended_abstract_DeChiara.pdf 
Dinardo, S. (2013). Guidelines for the SAR (Delay-Doppler) L1b Processing. Esrin, ESA, Italy. Available at: www.esa.int

Gelsthorpe R.V., Schied E., \& Wilson, J. J. W. (2000). ASCAT - MetOp's Advanced Scatterometer. ESA Bulletin, 102, 19-27.

GlobeSAR Program, Canada Centre for Remote Sensing, Natural Resources Canada. Available at: http://www.ccrs.nrcan.gc.ca/ccrs/rd/programs/globsar/gsarmain_e.html

Jenkins, F. A., \& White, H. E. (1957). Fundamentals of Optics. New York: McGraw-Hill.

Klauder J. R., Price, A. C., Darlington, S, \& Alversheim, W. J. (1960). The theory and design of Chirp Radars. The Bell System Technical Journal, XXXIX (4). American Telephone and Telegraph Company.

Kurtin O. D. (2013). Satellite Life Extension: Reaching for the Holy Grail. Publications, Via Satellite. New York: Kurtin PLLC.

Lillesand, T. M., Kiefer, R. W., \& Chipman, J. W. (2004). Remote Sensing and Image Interpretation. New York: John Wiley.

Oki R., Kubota, T., Katagiri, S., Kachi, M., Shimizu, S., Kojima, M., \& Kimura, K. (2010). Cloud and Precipitation Observation by Spaceborne Radar in Japan: Current and Future Missions. International Archives of the Photogrammetry, Remote Sensing and Spatial Information Science, Volume XXXVIII, Part 8, Kyoto, Japan.

Rosmorduc, V., Benveniste, J., Bronner, E., Dinardo, S., Lauret, O., Maheu, C., Milagro, M., \& Picot, N. (2011). In J. Benveniste \& N. Picot (Eds.), Radar Altimetry Tutorial. Available at: Available at: http://www.altimetry.info/documents/Radar_Altimetry_Tutorial_20110216.pdf

Rossi, B. B. (1957). Optics. Reading, Massachusetts: Addison-Wesley.

Sabins, F. F. (1999). Remote Sensing: Principles and Interpretation. New York: W. H. Freeman \& Co.

Wang J., \& Wolff, D. B. (2009). Evaluation of TRMM Ground-Validation Radar-Rain Errors Using Rain Gauge Measurements. Journal of Applied Meteorology and Climatology, 49, 310-324.

World Meteorological Organization (WMO). (2008). Guide to Meteorological Instruments and Methods of Observation. WMO-No. 8 (seventh edition). 